\title{
An Enhanced Fractional Order Model of Ionic Polymer-Metal Composites Actuator
}

\author{
R. Caponetto, S. Graziani, F. Sapuppo, and V. Tomasello \\ Dipartimento di Ingegneria Elettrica Elettronica ed Informatica, University of Catania, Viale A. Doria 6, 95125 Catania, Italy \\ Correspondence should be addressed to R. Caponetto; riccardo.caponetto@dieei.unict.it
}

Received 11 May 2013; Accepted 17 July 2013

Academic Editor: J. A. Tenreiro Machado

Copyright (C) 2013 R. Caponetto et al. This is an open access article distributed under the Creative Commons Attribution License, which permits unrestricted use, distribution, and reproduction in any medium, provided the original work is properly cited.

Ionic polymer-metal composites (IPMCs) are electroactive polymers which transform the mechanical forces into electric signals and vice versa. The paper proposes an enhanced fractional order transfer function (FOTF) model for IPMC membrane working as actuator. In particular the IPMC model has been characterized through experimentation, and a more detailed structure of its FOTF has been determined via optimization routines. The minimization error was attained comparing the simple genetic algorithms with the simplex method and considering the error between the experimental and model derived frequency responses as cost functions.

\section{Introduction}

In the last decade a new breed of polymers, known as electroactive polymers or more commonly EAPs, has emerged thanks to their electroactive capabilities [1]. Ionic polymermetal composites [2] belong to this material class. They bend if they are solicited by an external electric field and they act as motion sensor if an external deformation is applied. They are characterized by several interesting properties such as high compliance, lightness, and softness. IPMCs exploit ionic polymers for electrochemical and mechanical transduction and noble metals as electrodes, and they represent a valid alternative to the classic actuators and/or sensors. They can be cut in any shape and size, they are characterized by large deformations applying very low level of voltage, and they can work both in a humid or in a wet environment. These properties make them particularly attractive for possible applications in very different fields such as robotics, aerospace, and biomedicine $[3,4]$.

An intense research has been currently carried out to improve the IPMC performances in terms of power consumption, developed force, and deformation [5].

Moreover the research efforts have been spent in finding improved models able to predict the IPMCs behavior both as actuators and sensors [6].
In the literature several models describing the IPMC as actuator can be found. In detail, they can be divided into three categories: white box, black box, and grey box.

The first category, called white box, or physical models, is based on the underlying physical mechanisms of the IPMC to develop a system of equations that fully describes the device response [7]. Numerical implementation via methods as finite element analysis can be found in the literature [8]. Some difficulties are presented with physical modeling of IPMC transducers, such as a complete knowledge of the chemical and/or physical mechanisms involved in the electromechanical transduction and the direct measurement of some material parameters; moreover the numerical implementations are computationally onerous due to the distributed nature of the problem solution.

The second approach for modeling IPMC actuators is called black box and uses a linear [9] or differential [10] equation to simulate the actuating behavior. Such models, called also empirical and phenomenological, are based on the identification of coefficients through a series of curve fits based on the experimental data. The internal physics is, in this case, just a minor consideration.

An alternative to the complicated physical models and the simplistic and not scalable empirical models is the grey-box models. They comprise the fundamental physical laws into 
time-domain equations or transfer functions and empirically identified parameters to describe IPMC electromechanical behavior [11].

The grey-box model identification is often a multiobjective optimization problem in a multidimensional space since multiple cost functions can be used for parameters optimal estimation.

In a previous work, see [12], the authors have already proposed a fractional grey-box model of an IPMC actuator. In the present paper two novelties, with respect to [12], are introduced. The first one is related to the data acquisition. In fact instead of using, as input, a chirp signal it was applied a step-by-step frequency sweep. The second one is related to the structure of the interpolating FOTF; in this paper a further term has been added to the FOTF, obtaining a more accurate measurement fitting.

A very popular method in the field of parameter identification is the Nelder-Mead unconstrained simplex algorithm [13]. The method works on the exploration of the design space and does not require any derivative information, being therefore suitable for problems with nonsmooth functions. It is widely used in optimization software tools (i.e., MATLAB) to solve parameter estimation and similar statistical problems, when the function values come from experimentation and are thus uncertain or subject to noise [14]. On the other hand, the simplex method is prone to local minima issues, and the lack of convergence theory is often reflected in practice as a numerical breakdown of the algorithm, even for smooth and well-behaved functions.

Genetic algorithms (GAs), that explore a poorly understood solution space in parallel by intelligent trials, represent a class of optimization procedures able to face nonconvex optimization problem and to provide optimal solution avoiding remaining trapped in local minima $[15,16]$.

In the following, the two methods have been applied and compared in order to optimize the parameters of the interpolating FOTF.

The paper is structured as follows. Some introductive notes on fractional order systems are given in Section 2. A view on IPMC physics and working principles is presented in Section 3 in order to give an introduction on such a composite material; moreover the description of the experimental setup is given as the basis for understanding the experimental data used for model identification. In Section 4 the optimized FOTFs of the IPMC membrane are given, and finally some conclusions are reported.

\section{Fractional Order System}

The subject of fractional order calculus or noninteger order systems, that is, the calculus of integrals and derivatives of any arbitrary real or complex order, has gained considerable popularity and importance during the last three decades with applications in numerous seemingly diverse and widespread fields of science and engineering [17-19].

Fractional derivatives provide an excellent tool for the description of memory and hereditary properties of various materials and processes. This is the main advantage of fractional derivatives in comparison with classical integer-order models, in which such effects are in fact neglected. The advantages of fractional derivatives become apparent in modeling mechanical and electrical properties of real materials.

The most frequently used definition for the general fractional differintegral is the Caputo one, see [19]:

$$
{ }_{a} D_{t}^{r} f(t)=\frac{1}{\Gamma(r-n)} \int_{a}^{t} \frac{f^{(n)}(\tau)}{(t-\tau)^{r-n+1}} d \tau,
$$

for $(n-1<r<n)$. The initial conditions for the fractional order differential equations with the Caputo derivatives are in the same form as for the integer-order differential equations.

In the above definition, $\Gamma(m)$ is the factorial function, defined for positive real $m$, by the following expression:

$$
\Gamma(m)=\int_{0}^{\infty} e^{-u} u^{m-1} d u .
$$

Also for fractional order systems it is possible to apply the Laplace transformation. It assumes the form

$$
L\left\{\frac{d^{q} f(t)}{d t^{q}}\right\}=s^{q} L\{f(t)\}-\sum_{k=0}^{n-1} s^{k}\left[\frac{d^{q-1-k} f(t)}{d t^{q-k-1}}\right]_{t=0}
$$

and allows to easily manage fractional differential equation as noninteger order transfer function.

The fractional order transfer function of incommensurate real orders assumes the following form [18]:

$$
G(s)=\frac{b_{m} s^{\beta_{m}}+\cdots+b_{1} s^{\beta_{1}}+b_{0} s^{\beta_{0}}}{a_{n} s^{\alpha_{n}}+\cdots+a_{1} s^{\alpha_{1}}+a_{0} s^{\alpha_{0}}},
$$

where $a_{k}(k=0, \ldots, n), b_{k}(k=0, \ldots, m)$ are constant and $\alpha_{k}$ $(k=0, \ldots, n), \beta_{k}(k=0, \ldots, m)$ are arbitrary real or rational numbers, and without loss of generality they can be arranged as $\alpha_{n}>\alpha_{n-1}>\cdots>\alpha_{0}$ and $\beta_{m}>\beta_{m-1}>\cdots>\beta_{0}$.

Since in this case the values of fractional exponents need to be estimated along with the corresponding transfer function coefficients adequate optimization procedures need to be used.

\section{IPMC: Physics and Experimental Configuration}

3.1. Working Principles and Manufacturing. IPMC consists of a fluorocarbon membrane containing sulfonate groups covered on both sides with a thin noble metal coating layer. The IPMC actuator sample is manufactured with three primary coatings and one secondary coating of platinum. To increase platinum deposition the dispersing agent polyvinylpyrrolidone (PVP) has been used with a concentration of $0.001 \mathrm{M}$ [2]. The core of device is based on Nafion, distributed by DuPont; the characteristics of such an ionic polymer working in a humid environment allow the IPMC to work as actuator. The liquid molecules (generally water) which are mobile in the polymer structure, in fact, are at the basis for the electrochemical and mechanical transduction; they are driven by the voltage applied to the polymer via the metallic electrodes. The metal used to realize the device electrodes is usually platinum or gold. 


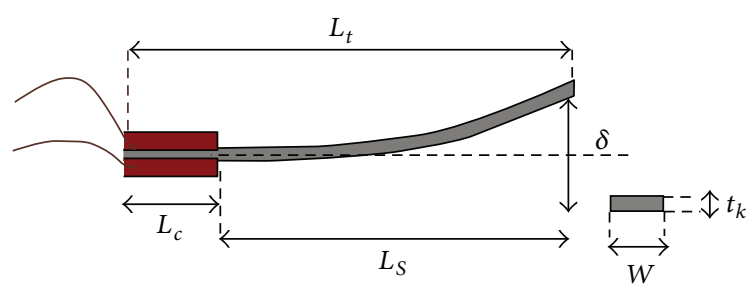

FIGURE 1: IPMC structure.

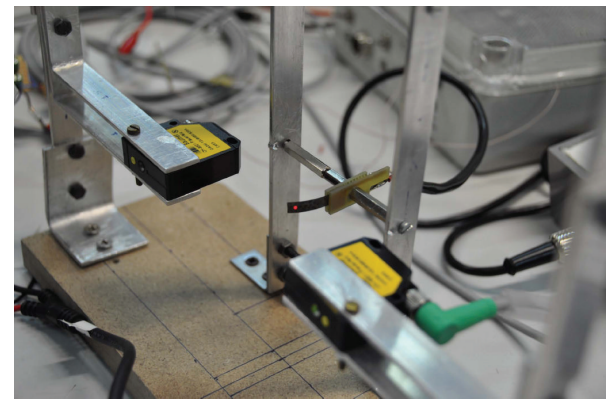

FIgURE 2: Experimental setup: mechanical structure, IPMC membrane, and laser beam.

3.2. Experimental Setup. The IPMC model was developed considering a configuration of a beam clamped at one end, as schematized in Figure 1. The pinned end is also used to apply the electrical stimulus via the electrodes.

The geometric parameters of the IPMC sample, as reported in Figure 1, are $L_{S}=28 \mathrm{~mm}, L_{C}=6 \mathrm{~mm}, t_{k}=$ $200 \mu \mathrm{m}$, and $W=5 \mathrm{~mm}$.

While the input signals $V_{\text {in }}$ were applied to the IPMC electrodes, the free deformation $\delta$ has been measured through a laser distance sensor, Baumer Electric OADM 12.

In order to determine the IPMC frequency response, sinusoidal inputs at different frequencies have been applied.

The considered range is between $50 \mathrm{mHz}$ and $50 \mathrm{~Hz}$ with an amplitude of $3 \mathrm{Vpp}$, and the following set of frequencies has been applied:

$$
\text { frequency }=\left[\begin{array}{lllllllll}
0.05 & 0.1 & 0.4 & 0.7 & 1 & 3 & 7 & 10 & 13
\end{array}\right.
$$

$$
\begin{array}{lllllllll}
17 & 20 & 22 & 25 & 27 & 30 & 31 & 32 & 33
\end{array}
$$

$$
\begin{array}{lllllllll}
34 & 35 & 36 & 37 & 38 & 40 & 43 & 47 & 50]
\end{array} \text {. }
$$

Three acquisition sets have been considered, in the following referred as $\mathrm{Acq}_{1}, \mathrm{Acq}_{2}$, and $\mathrm{Acq}_{3}$.

Figure 3 shows the magnitude and the phases of the acquired signals. The three measurement sets have been successively acquired starting from the lower frequency towards the higher one and then back to the lower one.

The sampling frequency is the same for all the sinusoidal inputs and is equal to $1100 \mathrm{~Hz}$.

The mechanical structure gripping the IPMC membrane and the measurement laser are shown in Figure 2.
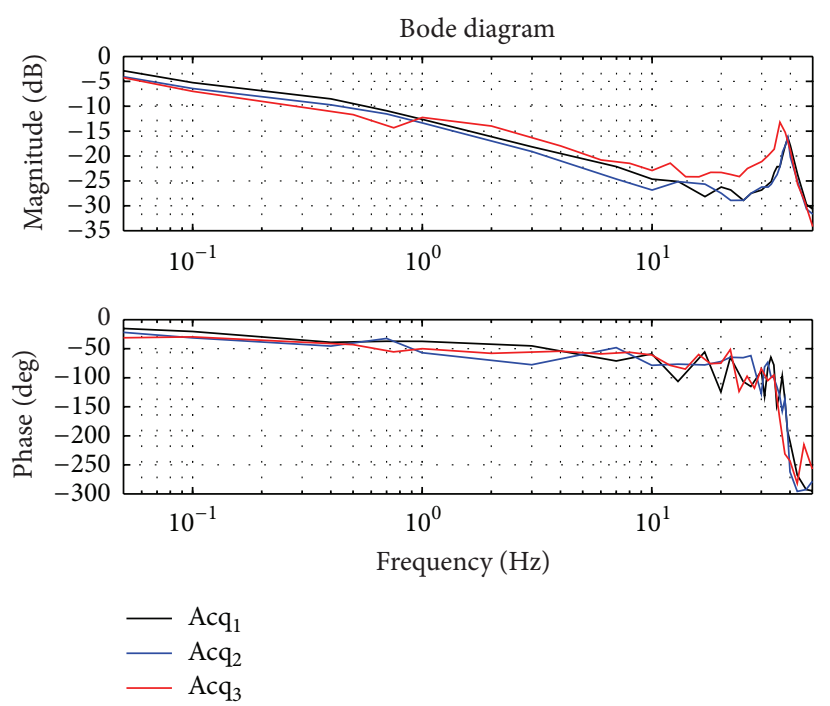

FIGURE 3: Magnitude and phase for the three acquisition sets.

\section{Fractional Order Transfer Function Modeling}

The acquired data shows a second-order like frequency response; see Figure 3. It is possible to note that, at low frequency, both the magnitude and the phase of the Bode diagram show a trend of fractional order.

It is possible to view that the magnitude curve slope is lower than $20 \mathrm{db} / \mathrm{dec}$, and the phase does not follow integer order variations.

The high-frequency noise that affects the frequency response is due to the low quality of the measured signals, and it mainly affects the phase representation.

These considerations suggest considering a fractional order model. The following building blocks for the FOTF have been therefore considered:
(i) $G_{1}(s)=k / s^{\alpha}$
(ii) $G_{2}(s)=1 /\left(1+\tau s^{\alpha 1}\right)$
(iii) $G_{3}(s)=w_{n}^{2} /\left(s^{2}+2 \xi w_{n} s+w_{n}^{2}\right)^{\alpha 2}$.

The first term allows to model a fractional pole at $s=0$, the second one a fractional order pole with time constant $\tau$, and the third one a second order fractional order term with a pair of complex poles.

The structure of the complete model for the FOTF if assumed is as follows:

$$
G(s)=G_{1}(s) G_{2}(s) G_{3}(s) .
$$

Applications of IPMC as actuators imply the availability of a good model at low frequency.

The model representation in (6) proves to be a more accurate model at low frequencies with respect to the one presented in [12].

Taking into account the sets of available measurements, four different frequency models have been determined. 
TABLE 1: FOTF parameters GAs optimized.

\begin{tabular}{|c|c|c|c|c|c|c|c|c|}
\hline & $k$ & $\alpha$ & $\tau$ & $\alpha_{1}$ & $\alpha_{2}$ & $w_{n}$ & $\xi$ & Error \\
\hline Model $_{1}$ on $\mathrm{Acq}_{1}$ data & 3.000 & 0.181 & 0.766 & 0.783 & 1.281 & 40.273 & 0.079 & $21.38 \%$ \\
\hline Model $_{2}$ on $\mathrm{Acq}_{2}$ data & 4.21 & 0.184 & 1.216 & 0.695 & 1.320 & 39.233 & 0.079 & $21.53 \%$ \\
\hline $\mathrm{Model}_{3}$ on $\mathrm{Acq}_{3}$ data & 1.878 & 0.268 & 0.392 & 0.663 & 1.261 & 37.876 & 0.120 & $22.18 \%$ \\
\hline Mean error model & 6.756 & 0.225 & 0.847 & 0.668 & 1.401 & 39.867 & 0.110 & $21.11 \%$ \\
\hline
\end{tabular}

TABLE 2: FOTF parameters simplex optimized.

\begin{tabular}{|c|c|c|c|c|c|c|c|c|}
\hline & $k$ & $\alpha$ & $\tau$ & $\alpha_{1}$ & $\alpha_{2}$ & $w_{n}$ & $\xi$ & Error \\
\hline Model $_{1}$ on $\mathrm{Acq}_{1}$ data & 0.693 & 0.330 & 0.257 & 0.810 & 1.132 & 40.064 & 0.059 & $21.81 \%$ \\
\hline $\mathrm{Model}_{2}$ on $\mathrm{Acq}_{2}$ data & 1.374 & 0.300 & 0.499 & 0.700 & 1.222 & 39.175 & 0.066 & $21.45 \%$ \\
\hline $\mathrm{Model}_{3}$ on $\mathrm{Acq}_{3}$ data & 2.835 & 0.225 & 0.587 & 0.612 & 1.300 & 36.916 & 0.107 & $23.25 \%$ \\
\hline Mean error model & 0.482 & 0.376 & 0.196 & 0.798 & 1.101 & 39.536 & 0.055 & $22.82 \%$ \\
\hline
\end{tabular}

The first three are obtained from the measurement sets $\mathrm{Acq}_{i}$, while the fourth one is determined as a model with a mean error computed over the three measurement sets.

The parameters of the transfer function $G(s)$ that are $k, \alpha$, $\tau, \alpha_{1}, \alpha_{2}, w_{n}$, and $\xi$ have been determined applying both the simplex method and GAs.

The object function applied during the optimization procedures takes into account both the module and the phase of the FOTF and consists in the sum of two terms:

$$
\mathrm{OBJ} 1=\frac{\sqrt{\sum\left(G_{\text {meas }}-G_{\text {sim }}\right)^{2}}}{\sqrt{\sum G_{\text {meas }}^{2}}},
$$

where $G_{\text {meas }}$ is the gain of the measured signal and $G_{\text {sim }}$ is the module of the simulated one.

And the second term takes into account the phase

$$
\mathrm{OBJ} 2=\frac{\sqrt{\sum\left(\mathrm{Ph}_{\text {meas }}-\mathrm{Ph}_{\text {sim }}\right)^{2}}}{\sqrt{\sum \mathrm{Ph}_{\text {meas }}^{2}}},
$$

where $\mathrm{Ph}_{\text {meas }}$ is the phase of the measured signal and $\mathrm{Ph}_{\text {sim }}$ is the phase of the simulated one.

Tables 1 and 2 report the results related to the FOTF parameters identification applying GAs and simplex method, respectively.

The Bode diagrams of the obtained FOTF are given in Figures 4, 5, 6, 7, 8, 9, 10, and 11 .

Figures 4-7 show the frequency responses obtained via GAs while Figures 8-11 via simplex method.

According to the error provided in Tables 1 and 2 both the optimization procedures provide a good frequency matching.

It is worth noticing that the models obtained via GAs (Figures 4-7) provide a better fitting at low frequencies.

\section{Conclusion}

The paper proposes an enhanced fractional order transfer function model of an IPMC membrane working as actuator. The IPMC model has been determined exploiting experimental data.
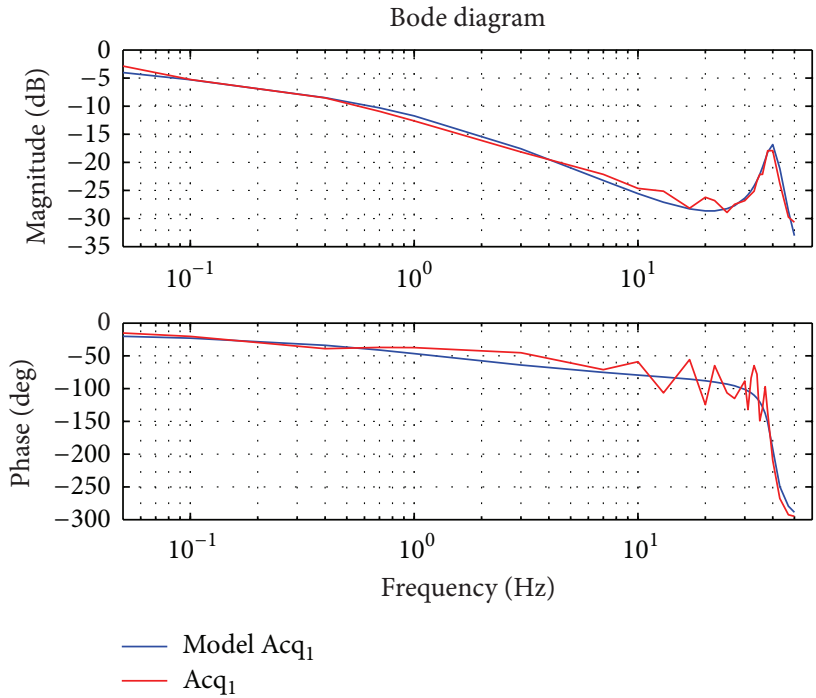

Figure 4: Magnitude and phase of the model on $\mathrm{Acq}_{1}$ data via GAs.
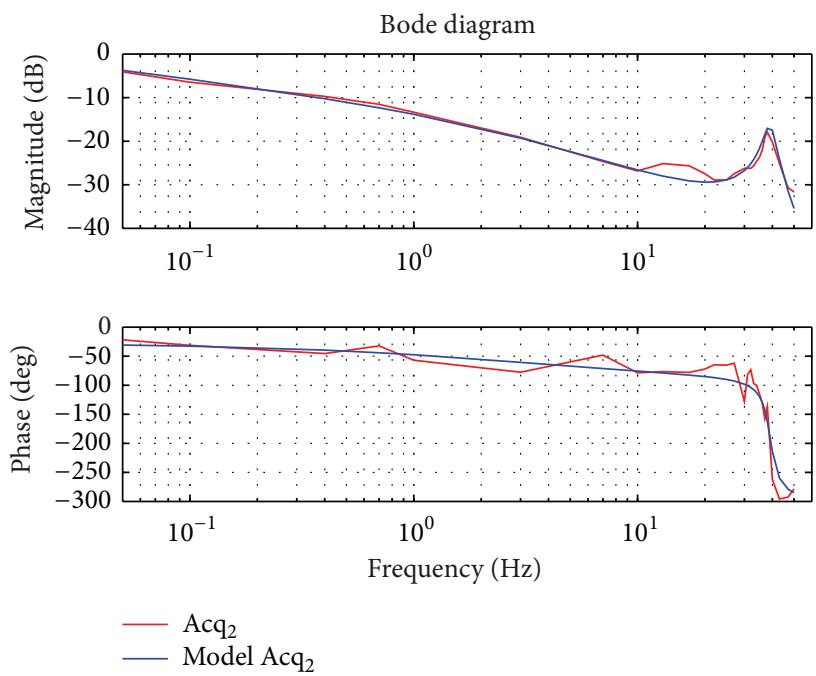

Figure 5: Magnitude and phase of the model on $\mathrm{Acq}_{2}$ data via GAs. 

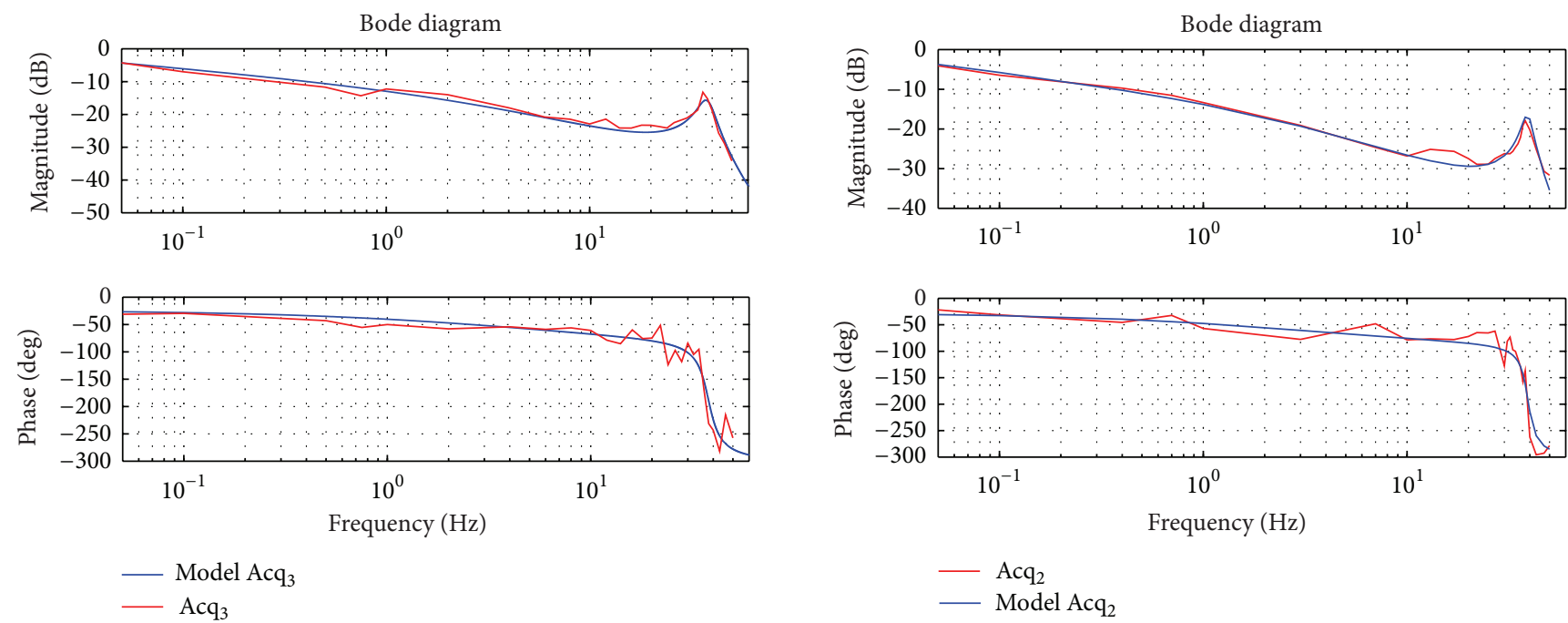

Figure 6: Magnitude and phase of the model on $\mathrm{Acq}_{3}$ data via GAs.

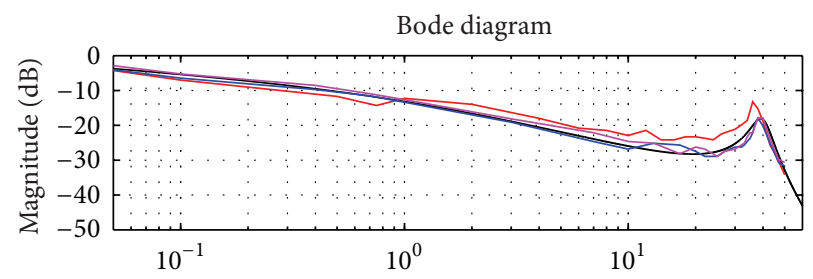

Figure 9: Magnitude and phase of the model on $\mathrm{Acq}_{2}$ data via simplex method.
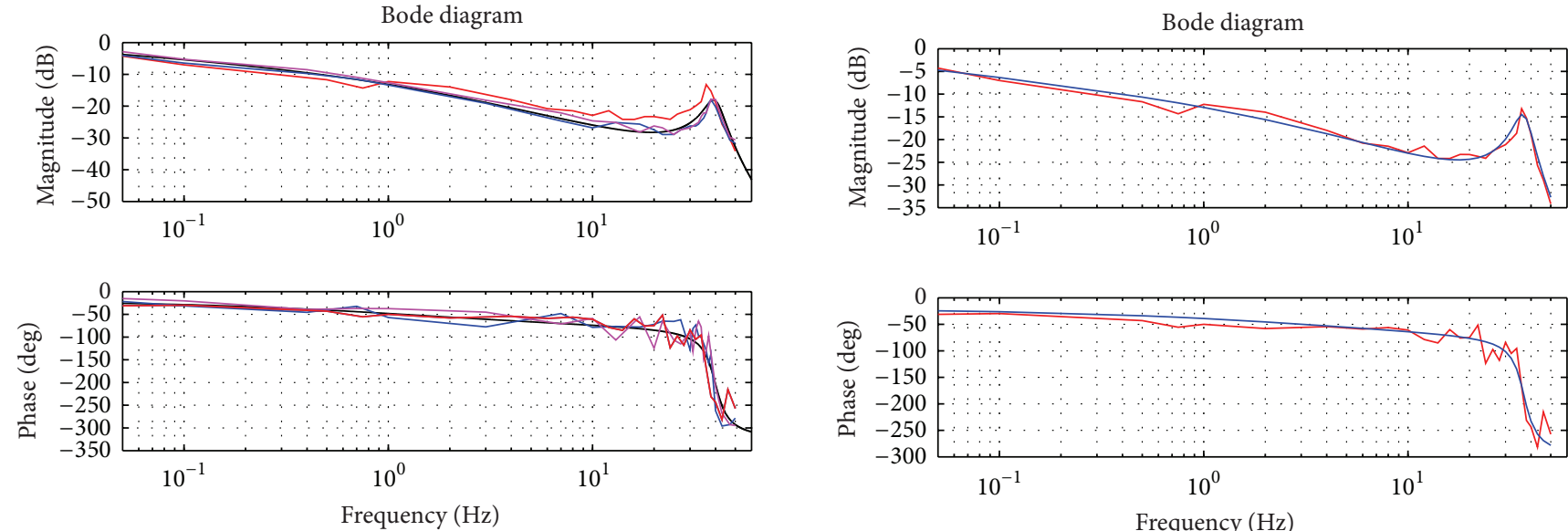

$$
\begin{aligned}
& \text { Medium model } \\
& -\mathrm{Acq}_{3} \mathrm{Acq}_{2} \\
& -\mathrm{Acq}_{1}
\end{aligned}
$$

Figure 7: Magnitude and phase of the mean model on $\mathrm{Acq}_{1}, \mathrm{Acq}_{2}$, and $\mathrm{Acq}_{3}$ via GAs.
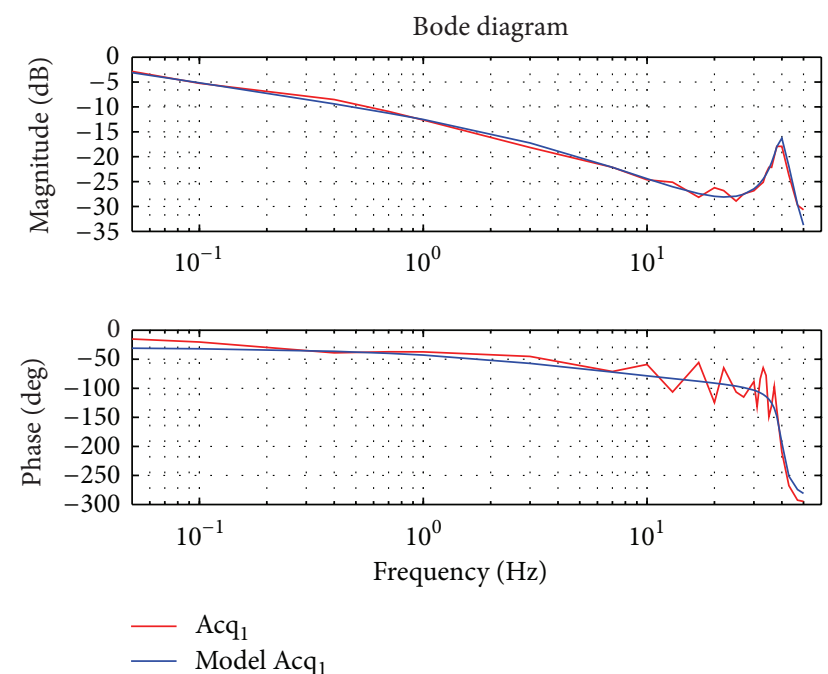

FIgURE 8: Magnitude and phase of the model on $\mathrm{Acq}_{1}$ data via simplex method.

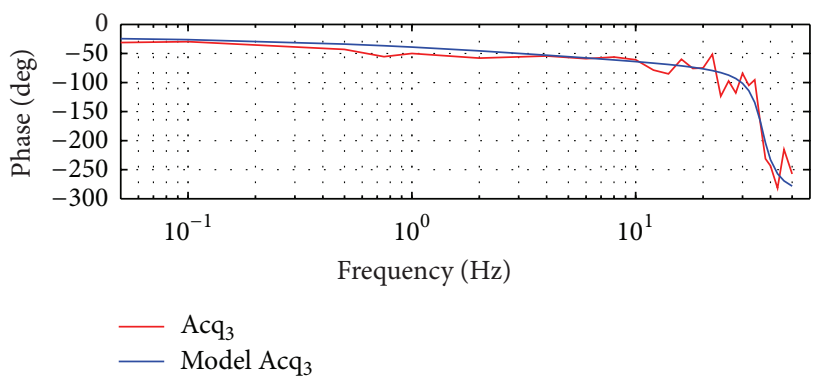

Figure 10: Magnitude and phase of the model on $\mathrm{Acq}_{3}$ data via simplex method.
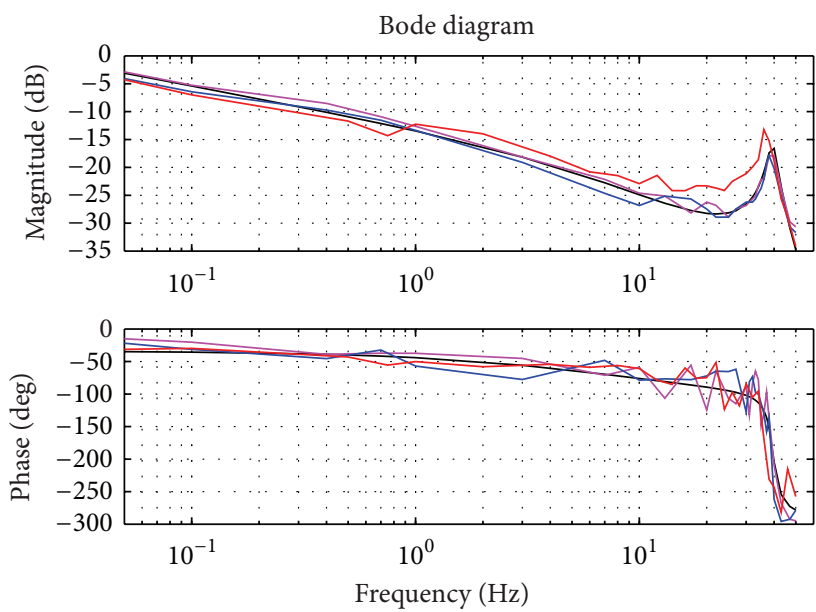

$$
\text { - Mean error model } \quad-\mathrm{Acq}_{2}
$$

FIGURE 11: Magnitude and phase of the mean model on $\mathrm{Acq}_{1}, \mathrm{Acq}_{2}$, and $\mathrm{Acq}_{3}$ via simplex method. 
The model was proven to be very accurate at low frequencies, and such frequency matching makes it suitable for control system design.

\section{Acknowledgment}

This work has been supported by the Italian Ministry of University and Research (MIUR) under PRIN projects "Noninteger order systems in modeling and control," Grant no. 2009F4NZJP.

\section{References}

[1] Y. Bar-Cohen, "Electro-active polymers: current capabilities and challenges," in Proceedings of the SPIE Smart Structures and Materials Symposium, EAPAD Conference, pp. 4695-4702, San Diego, Calif, USA, 2002.

[2] M. Shahinpoor and K. J. Kim, "Ionic polymer-metal composites-I. Fundamentals," Smart Materials and Structures, vol. 10, no. 4, pp. 819-833, 2001.

[3] M. Shahinpoor and K. J. Kim, "Ionic polymer-metal composites-4. Industrial and medical applications," Smart Materials and Structures, vol. 14, no. 1, pp. 197-214, 2005.

[4] C. Bonomo, P. Brunetto, L. Fortuna, P. Giannone, S. Graziani, and S. Strazzeri, "A tactile sensor for biomedical applications based on IPMCs," IEEE Sensors Journal, vol. 8, no. 8, pp. 14861493, 2008.

[5] A. Punning, M. Anton, M. Kruusmaa, and A. Aabloo, "An engineering approach to reduced power consumption of IPMC (Ion-Polymer Metal Composite) actuators," in Proceedings of the 12th International Conference on Advanced Robotics (ICAR '05), pp. 856-863, July 2005.

[6] D. J. Leo, K. Farinholt, and T. Wallmersperger, "Computational models of ionic transport and electromechanical transduction in ionomeric polymer transducers," in Proceedings of the Smart Structures and Materials, Electroactive Polymer Actuators and Devices (EAPAD '05), pp. 170-181, March 2005.

[7] S. Nemat-Nasser and J. Y. Li, "Electromechanical response of ionic polymer-metal composites," Journal of Applied Physics, vol. 87, no. 7, pp. 3321-3331, 2000.

[8] R. Caponetto, V. De Luca, S. Graziani, F. Sapuppo, and E. Umana, "A multi-physics model of an IPMC actuator in the electrical, chemical, mechanical and thermal domains," in Proceedings of the SMACD, Seville, Spain, 2012.

[9] R. Kanno, A. Kurata, and M. Hattori, "Characteristics and modeling of ICPF actuator," in Proceedings of the Japan-USA Symposium on Flexible Automation, vol. 2, pp. 691-698, 1994.

[10] Y. Xiao and K. Bhattacharya, "Modeling electromechanical properties of ionic polymers," in Proceedings of the Electroactive Polymer, Actuators and Devices-Smart Structures and Materials, pp. 292-300, March 2001.

[11] P. Brunetto, L. Fortuna, P. Giannone, S. Graziani, and S. Strazzeri, "Static and dynamic characterization of the temperature and humidity influence on IPMC actuators," IEEE Transactions on Instrumentation and Measurement, vol. 59, no. 4, pp. 893-908, 2010.

[12] R. Caponetto, G. Dongola, L. Fortuna, S. Graziani, and S. Strazzeri, "A fractional model for IPMC actuators," in Proceedings of the IEEE International Instrumentation and Measurement Technology Conference, pp. 2103-2107, May 2008.
[13] J. A. Nelder and R. Mead, "A simplex method for function minimization," The Computer Journal, vol. 7, no. 4, pp. 308-313, 1965.

[14] J. C. Lagarias, J. A. Reeds, M. H. Wright, and P. E. Wright, "Convergence properties of the Nelder-Mead simplex method in low dimensions," SIAM Journal on Optimization, vol. 9, no. 1, pp. 112-147, 1999.

[15] D. E. Goldberg, Genetic Algorithms in Search, Optimization and Machine Learning, Addison Wesley, 1989.

[16] Z. Michalewicz, Genetic Algorithms + Data Structures = Evolution Programs, Springer, Berlin, Germany, 2nd edition, 1994.

[17] K. B. Oldham and J. Spanier, The Fractional Calculus: Theory and Applications of Differentiation and Integration to Arbitrary Order, Dover Books on Mathematics, 2006.

[18] I. Podlubny, Fractional Differential Equations, vol. 198, Academic Press Inc., San Diego, CA, 1999.

[19] R. Caponetto, G. Dongola, L. Fortuna, and I. Petras, Fractional Order Systems: Modelling and Control Applications, vol. 72, Nonlinear Science, Series A, 2010. 


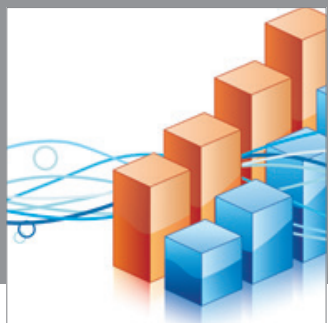

Advances in

Operations Research

mansans

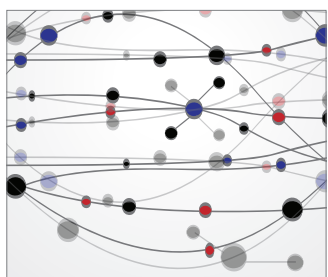

The Scientific World Journal
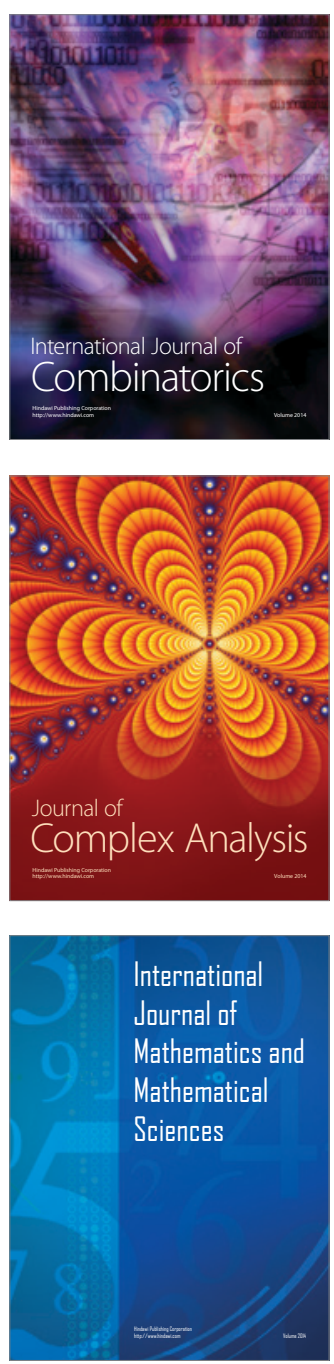
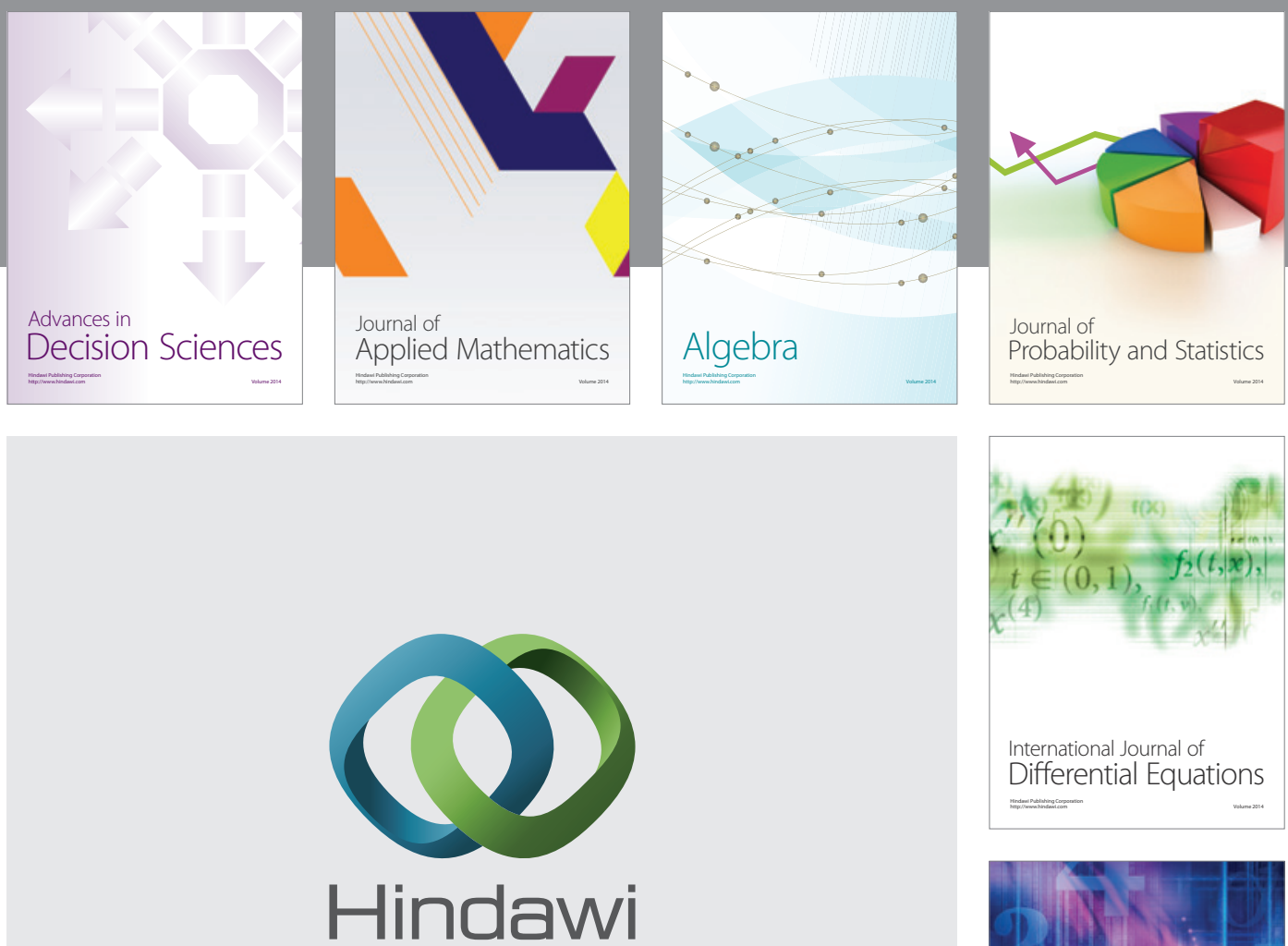

Submit your manuscripts at http://www.hindawi.com
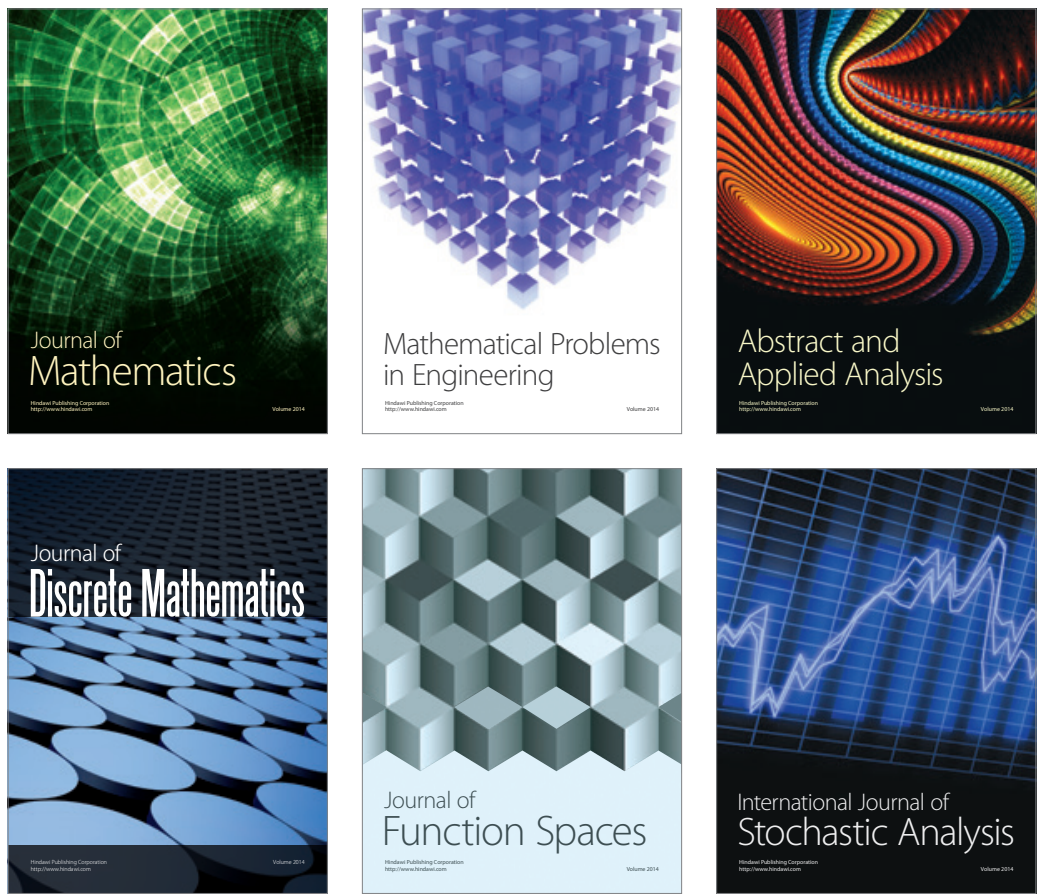

Journal of

Function Spaces

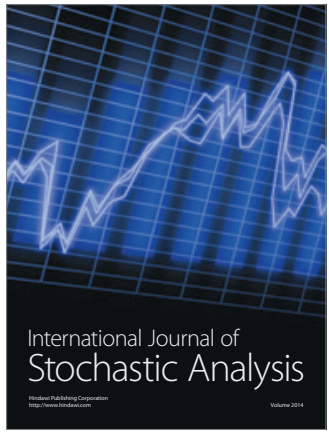

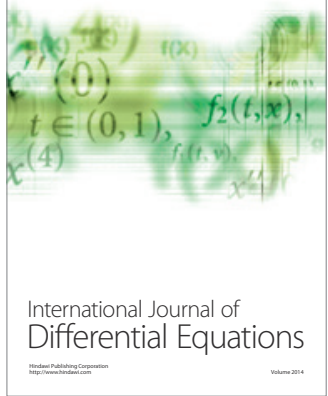
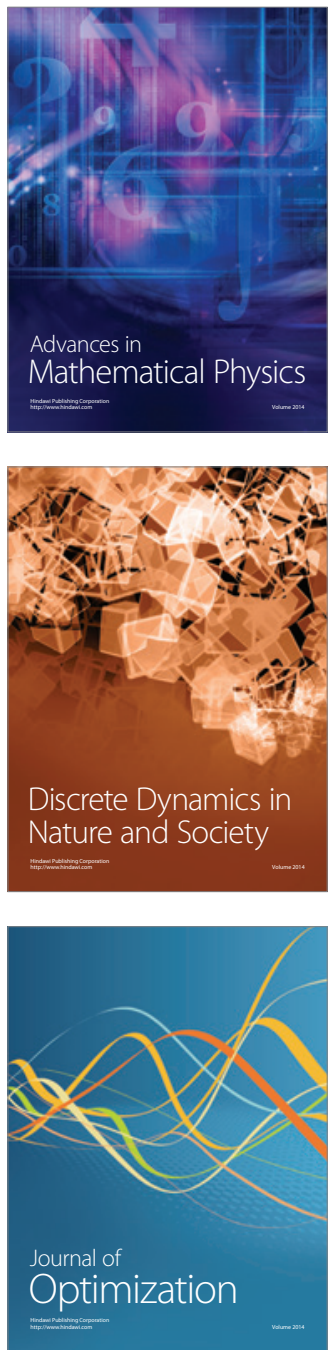